\title{
Experimental validation of opto-thermo-elastic modeling in OOFELIE Multiphysics
}

\author{
Alexandra Mazzoli*a, Philippe Saint-Georges ${ }^{\mathrm{b}}$, Anne Orban ${ }^{\mathrm{a}}$, Jean-Sébastien Ruess ${ }^{\mathrm{c}}$, Jérôme Loicq ${ }^{\mathrm{a}}$, \\ Christian Barbier ${ }^{\mathrm{a}}$, Yvan Stockman ${ }^{\mathrm{a}}$, Marc Georges $^{\mathrm{a}}$, Philippe Nachtergaele ${ }^{\mathrm{b}}$, Stéphane Paquay ${ }^{\mathrm{b}}$, \\ Pascal De Vincenzo ${ }^{b}$ \\ ${ }^{a}$ Centre Spatial de Liège, Av. Du Pré-Aily, 4031 Angleur, Belgium ; \\ ${ }^{\mathrm{b}}$ Open Engineering, Rue des Chasseurs-Ardennais 8, 4031 Angleur, Belgium ; \\ ${ }^{c}$ GDTech, Rue des Chasseurs-Ardennais 8, 4031 Angleur, Belgium
}

\begin{abstract}
The objective of this work is to demonstrate the correlation between a simple laboratory test bench case and the predictions of the OOFELIE Multiphysics software in order to deduce modeling guidelines and improvements. For that purpose two optical systems have been analyzed. The first one is a spherical lens fixed in an aluminium barrel, which is the simplest structure found in an opto-mechanical system. In this study, material characteristics are assumed to be well known: BK7 and aluminium have been retained. Temperature variations between 0 and $+60{ }^{\circ} \mathrm{C}$ from ambient have been applied to the samples. The second system is a YAG laser bar heated by means of a dedicated oven.

For the two test benches thermo-elastic distortions have been measured using a Fizeau interferometer. This sensor measures wavefront error in the range of $20 \mathrm{~nm}$ to $1 \mu \mathrm{m}$ without physical contact with the opto-mechanical system. For the YAG bar, birefringence and polarization measurements have also been performed using a polarimetric bench.

The tests results have been compared to the predictions obtained by OOFELIE Multiphysics which is a simulation software dedicated to multiphysics coupled problems involving optics, mechanics, thermal physics, electricity, electromagnetism, acoustics and hydrodynamics. From this comparison modeling guidelines have been issued with the aim of improving the accuracy of computed thermo-elastic distortions and their impact on the optical performances.
\end{abstract}

Keywords: OOFELIE, Multiphysics, lens, YAG laser rod, thermo-elastic, interferometer, birefringence, polarization

\section{INTRODUCTION}

Taking benefit of more and more powerful computers, the industrial requirements impose numerical modeling of large and complex systems with a higher accuracy and a better fidelity.

In order to take up the new challenges of numerical simulation, a research project has been funded by the Walloon Region (Belgium). The Multi- $\Phi$ project takes advantage of a strong synergy between software editor companies, research centers, universities and industrial end-users based in Wallonia. The goal is to develop an innovative and integrated software solution which is able to efficiently process large scale models involving strongly coupled multiphysics interactions. This solution is based on the current simulation modules OOFELIE Multiphysics, SAMCEF Field and FINE Suite respectively developed by the software editors Open Engineering, Samtech and Numflo.

The Multi- $\Phi$ project offers improvement of the solution and experimental validation via several test cases in the fields of opto-thermo-mechanics and fluid-structure interaction. Two of them are presented in this paper: the first one concerns the heating and deformation of a lens inside a mount, the second one studies the thermal lensing in a YAG laser bar and the thermally generated strain-induced birefringence. Theoretical results are also provided.

The OOFELIE Multiphysics software is introduced here below. Then follow the description of the two test benches as well as the results of the performed tests. Finally the simulations results are presented and compared to the tests results.

*amazzoli@ulg.ac.be; www.csl.ulg.ac.be 


\section{OOFELIE MULTIPHYSICS}

OOFELIE Multiphysics combines mechanical, thermal, acoustics, piezoelectric, pyro-piezoelectric, electrostatic, Peltier and Seebeck effects, current conduction, circuits, computational fluid dynamics, fluid structure interaction, vibroacoustics, MEMS and electromagnetic features. Historically based on the Finite Element Method (FEM) ${ }^{1}$, the OOFELIE toolkit presently integrates other simulation schemes: Boundary Element Method (BEM), Finite Differences Method (FDM) and Extended Finite Element Method (X-FEM). Strong and weak physical couplings are available. Monolithic, staggered and sequential resolution schemes are also available and can be adapted by the user.

One particularity of OOFELIE is its openness to collaborative developments in a scientific or R\&D framework. Indeed, the OOFELIE Software Development Kit (SDK) provides a set of dynamic libraries for customization of the software. Taking advantage of Object Oriented Programming techniques, advanced components can be developed. Emphasis was placed on the simplicity in the introduction of user defined elements, material laws and resolution schemes. The users have thus the possibility to adapt the standard behavior of OOFELIE to their needs. Innovative functionalities can be added via a $\mathrm{C}++$ like programming language within the user interface SAMCEF Field ${ }^{1}$.

OOFELIE Multiphysics also offers a unique automated link to the optical ray-tracing software ZEMAX. Coupled analyses of opto-thermo-electro-mechanical interactions are so available. That covers a wide range of applications such as steering mechanisms based on piezoelectric or active/adaptive optics. The communication with ZEMAX is based on the DDE protocol on Windows (Dynamic Data Exchange). This automated in-memory exportation process of the computed optical surface deformations reduces human interventions in order to save time and to reduce the risk of loss of data integrity.

Part of the complete solution OOFELIE, the OOFELIE for Advanced Optics tool is also linked to ZEMAX and is dedicated to opto-thermo-mechanical analyses. Static and transient analyses can be performed. Combining ease of use and fastness, this simulation tool leads to high precision optical designs. The modeled optical systems may involve mechanical components such as mounts, stages, rails or more complex structures, which are built in the SAMCEF Field user interface. To guarantee well defined optical surfaces, the geometry of the optical elements can be exported from ZEMAX to SAMCEF Field by use of standard CAD formats. Furthermore, a verification process is performed for each optical surface before starting the computation. If the surface sag is not represented with a sufficient precision, OOFELIE corrects the mesh by use of the analytical equation which is automatically retrieved from ZEMAX. The correction that is made is then visualized in the user interface. This correction process is useful for very high precision optics.

As a result of the performed thermo-mechanical analysis, the deformations of the optical surfaces are automatically exported to ZEMAX. A new ZEMAX file is created in which each optical surface is modified by use of a regular grid of values or a Zernike polynomial decomposition. The surface sag distortion is always considered along the optical axis of each optical surface. The choice is let to the user to account for transverse nodal displacements or not. If the deformed configuration contains rigid body components (translations and/or rotations), those parameters are identified and can be introduced separately in the updated optical model by use of surface thickness and tilt/decenter parameters.

OOFELIE for Advanced Optics and the complete solution OOFELIE Multiphysics, combined with ZEMAX, allow many applications in the field of optical system design and optimization. For example, one can model complex and large telescopes, high precision cameras, satellite optics, positioners, piezoelectric actuators, deformable mirrors... In addition, new advanced features such as electrically and mechanically induced birefringence are currently under development.

\section{TEST BENCHES}

\subsection{Spherical lens}

The spherical lens is biconvex and made of BK7 with a diameter of $50 \mathrm{~mm}$ and a focal length of $200 \mathrm{~mm}$. Its mount is in black anodized aluminium and includes a retainer ring that securely holds the lens in place. Both lens and mount are commercially available. The lens in its mount is maintained in the claws of a five axes mount to allow an alignment of the lens in front of the interferometer. The lens is heated by means of two heaters glued on the mount. Thermocouples are placed on the mount to monitor the temperature during the test (See Figure 1). 


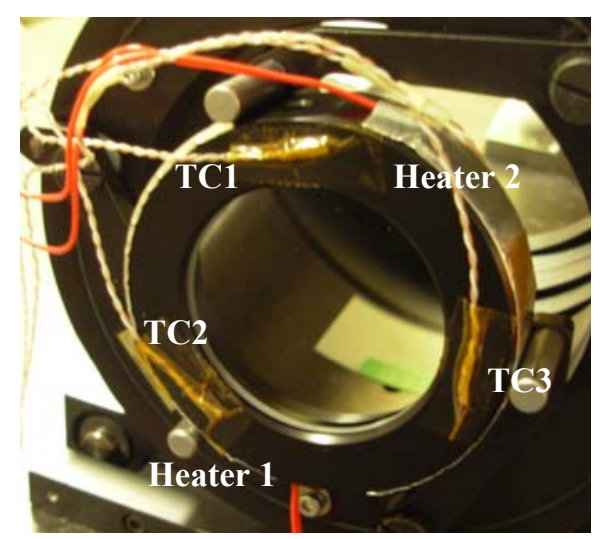

Figure 1. Distribution of heaters and thermocouples on lens mount

A spherical caliber with a F-number of 3.3 is placed at the output of the interferometer to use a converging beam (becoming diverging after its focus point) to fully illuminate the lens. The lens is placed at a distance to the focus point equal to its focal length in order to have a collimated beam after the lens. A plane mirror of high quality $(\lambda / 20)$ is used to send the collimated beam back towards the lens then into the interferometer. The optical sketch of this test is shown in Figure 2. The wavelength of the interferometer beam is $633 \mathrm{~nm}$.

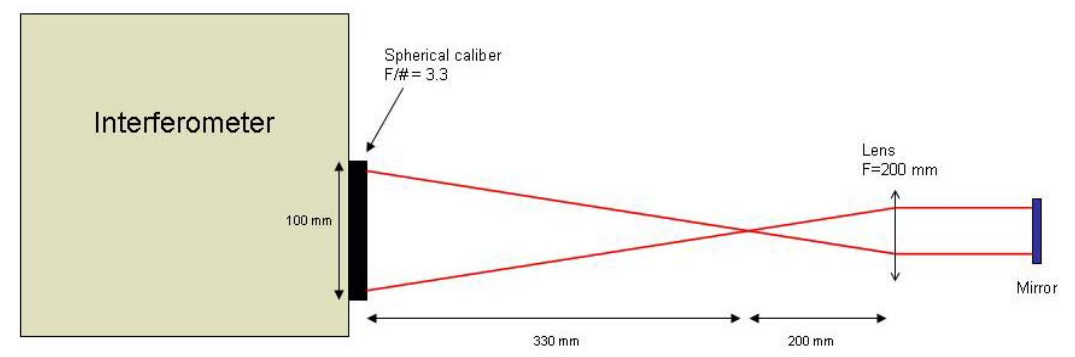

Figure 2. Optical sketch of the lens interferometric test

Temperature measurements with thermocouples glued on each face of the lens are first performed to determine the temperature in the center and at the edge of the lens as a function of the temperature on the mount. The thermocouples on the lens are then removed to allow the interferometric measurements. The latter ones are performed at ambient temperature and then for different temperatures when heating the mount. For each measurement we waited for the stabilization of the temperature on the thermocouples. The degradation of the optical performances of the lens is obtained by comparing the wavefront error (WFE) of the beam at warm temperature with the WFE at ambient temperature. A view of the test bench is shown in Figure 3.

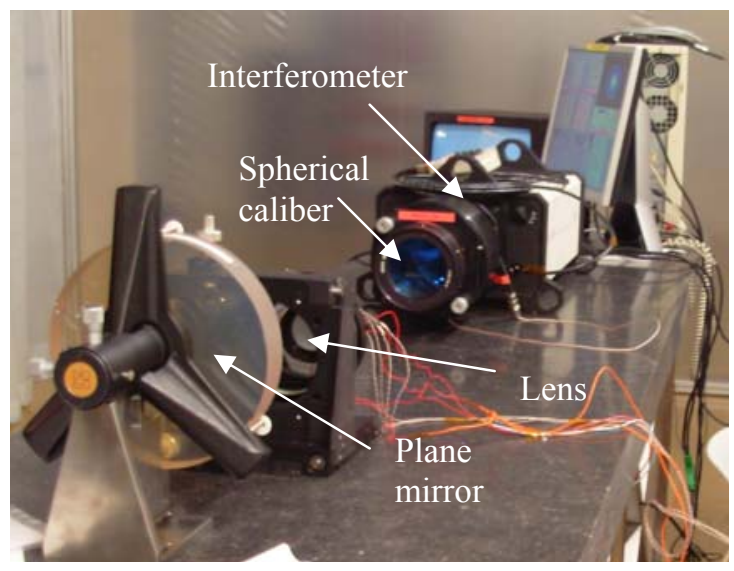

Figure 3. Spherical lens test bench 


\subsection{YAG bar}

The YAG bar presents a length of $50 \mathrm{~mm}$ and a square section of $12 \times 12 \mathrm{~mm}^{2}$. It is heated in a dedicated oven with a maximum allowed temperature of $200^{\circ} \mathrm{C}$ (See Figure 4).

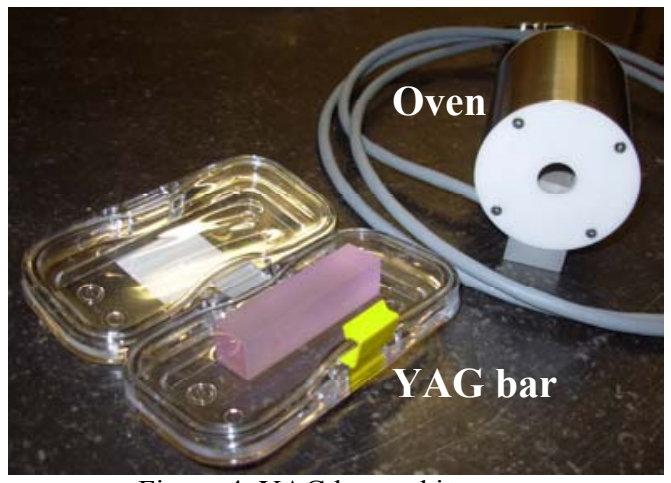

Figure 4. YAG bar and its oven

\subsubsection{Interferometric measurements}

For the interferometric measurements the YAG bar in its oven is placed between the interferometer and a plane mirror ( $\lambda / 20$ surface quality). The caliber at the output of the interferometer is a plane to produce a collimated beam. The wavelength of the beam is $633 \mathrm{~nm}$. The plane mirror is first aligned on the interferometer beam. The oven is placed on a rotation stage to allow the alignment of the YAG bar with the beam. A WFE measurement is first made at ambient temperature then the oven is heated and measurements are done for some temperatures between the ambient and $100^{\circ} \mathrm{C}$. A sketch of this test bench is presented in Figure 5.

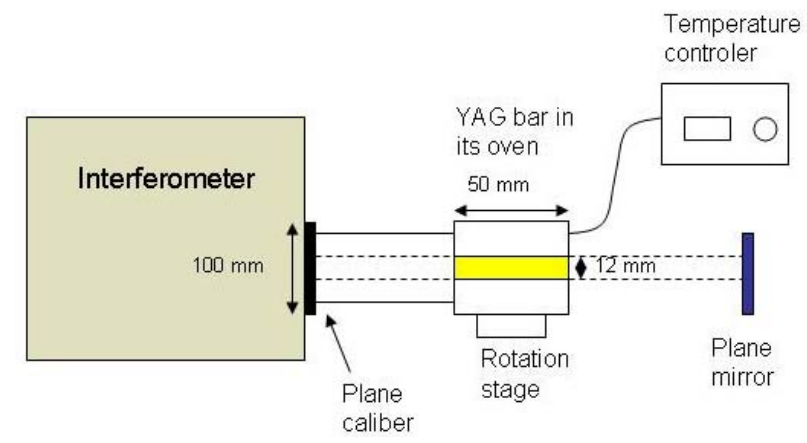

Figure 5. Sketch of the YAG bar interferometric test

\subsubsection{Polarimetric measurements}

A metrology bench for polarization measurements $(\lambda=633 \mathrm{~nm})$ has been set with accurate polarizers and acquisition data system. The measurement procedure has been thoroughly investigated and optimized. The setup and methodology of measurement are based on the ellipsometry technique. The methodology is the one of the rotating polarizer and fixed analyzer, as presented on Figure 6.

Qualification and reference measurements were successfully realized on $\lambda / 4$ wave-plate in order to validate the bench, the measurements and the data-analysis procedure.

For the polarimetric measurements the YAG bar in its oven is placed between two linear polarizers. Before the first polarizer a $\lambda / 4$ wave-plate is placed. A He-Ne laser beam is sent through all these elements until a detector. By rotating the second polarizer the birefringence can be measured. 


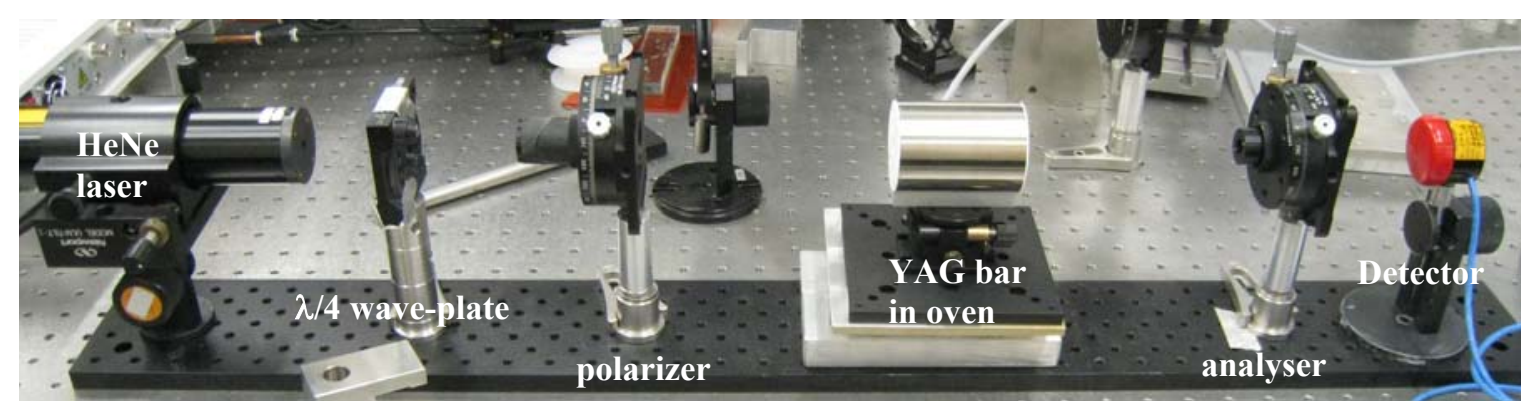

Figure 6. Polarimetric test bench

\section{TEST RESULTS}

\subsection{Spherical lens}

Figure 7 shows two examples of measured WFE. The first one corresponds to a measurement at ambient temperature and the second one to the maximum temperature applied which results in $58.4^{\circ} \mathrm{C}$ at the center of the lens. The third image is the result of the difference between both first WFE. The dominant aberration is focus and its impact on the lens properties is a modification of its focal length according to the formula ${ }^{4}$

$$
\Delta f=2 \cdot 4 F^{2} \cdot W F E \cdot \lambda
$$

where $\mathrm{F}$ is the f-number i.e. the focal length divided by the entrance pupil diameter, WFE is the part of the WFE corresponding to the focus and $\lambda$ is the wavelength.
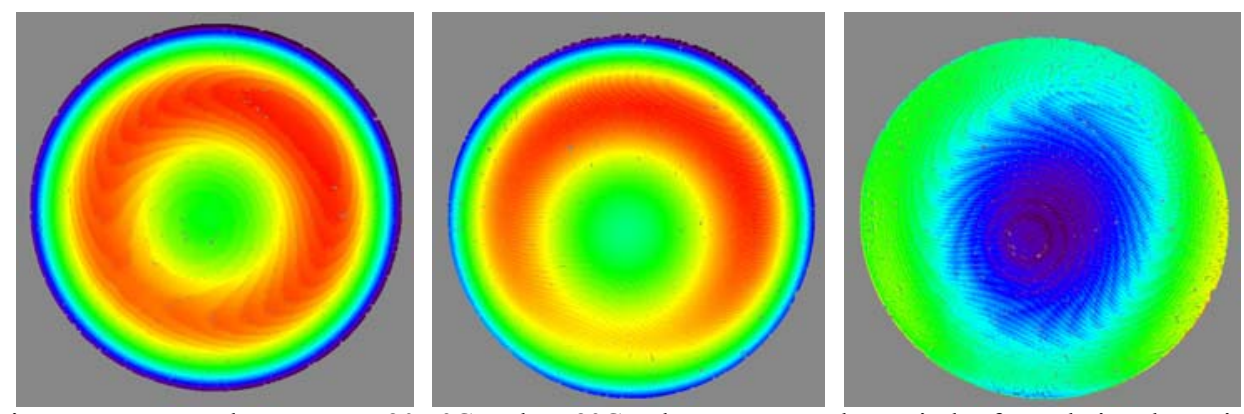

Figure 7. Measured WFE at $\mathrm{T}=20.5^{\circ} \mathrm{C}$ and $57.2^{\circ} \mathrm{C}$ at lens center and remainder from their subtraction

The results are summarized in Table 1 here below.

- The two first columns give the measured temperatures respectively on the lens mount and at the center of the lens.

- The third column presents the measured Zernike coefficients of the focus aberration from which the focus at ambient temperature has been subtracted. The fourth column is the WFE PV corresponding to this aberration and is equal to twice the absolute value of the focus Zernike coefficient.

- The fifth column is the variation of focal length due to the WFE of column 4 calculated with formula (1).

- The sixth column is the calculated focal length modification due to a temperature change in the lens $\Delta f=-x_{G}^{f} f \Delta T$ where $x_{G}^{f}$ is called the thermal coefficient of focal expansion and depends only on the lens glass. Its value is $-4.18810^{-6} /{ }^{\circ} \mathrm{C}$. 
- The WFE due to the gradient between the center and the edge of the lens is calculated following the formula $W F E=O P D \cdot \lambda=\left[\left(n_{G}-1\right)\left(1+v_{G}\right) \alpha_{G}+\frac{d n_{G}}{d T}\right] t \Delta T \cdot \lambda$ with the thickness $t=t_{0}\left(1+\alpha_{G} T\right)$ where $\mathrm{n}_{\mathrm{G}}$ is the refractive index of the glass, $v_{\mathrm{G}}$ is the Poisson coefficient of the glass and $\alpha_{\mathrm{G}}$ is the coefficient of thermal expansion of the glass $^{5,6}$. The last column is the variation of focal length due to this WFE calculated with formula (1).

Table 1. Summary of spherical lens test results

\begin{tabular}{|c|c|c|c|c|c|c|}
\hline $\begin{array}{c}\text { Average } \\
\text { temperature } \\
\text { on mount } \\
{\left[{ }^{\circ} \mathrm{C}\right]}\end{array}$ & $\begin{array}{c}\text { Temperature } \\
\text { at lens center } \\
{\left[{ }^{\circ} \mathrm{C}\right]}\end{array}$ & $\begin{array}{c}\text { Zernike } \\
\text { coefficient } \\
\text { of focus } \\
{[\lambda]}\end{array}$ & $\begin{array}{c}\text { Measured } \\
\text { WFE PV } \\
{[\lambda]}\end{array}$ & $\begin{array}{c}\text { Measured } \\
\text { thermal } \\
\text { defocus } \\
{[\mu \mathrm{m}]}\end{array}$ & $\begin{array}{c}\text { Calculated } \\
\text { thermal } \\
\text { defocus } \\
{[\mu \mathrm{m}]}\end{array}$ & $\begin{array}{c}\text { Calculated } \\
\text { defocus from } \\
\text { gradient } \\
{[\mu \mathrm{m}]}\end{array}$ \\
\hline 20.5 & 20.5 & 0.000 & 0.000 & 0.00 & 0.00 & 0.00 \\
36.8 & 31.0 & -0.107 & 0.213 & 18.74 & 8.79 & 8.93 \\
44.3 & 37.0 & -0.134 & 0.269 & 23.61 & 13.82 & 11.24 \\
58.6 & 46.5 & -0.236 & 0.471 & 41.44 & 21.78 & 18.63 \\
67.1 & 50.1 & -0.287 & 0.575 & 50.52 & 24.79 & 26.18 \\
78.7 & 58.4 & -0.390 & 0.780 & 68.54 & 31.74 & 30.80 \\
68.1 & 52.5 & -0.341 & 0.681 & 59.89 & 26.80 & 24.02 \\
59.3 & 46.8 & -0.258 & 0.515 & 45.29 & 22.03 & 19.25 \\
46.4 & 38.2 & -0.210 & 0.420 & 36.96 & 14.83 & 12.62 \\
39.9 & 34.0 & -0.122 & 0.244 & 21.45 & 11.31 & 9.08 \\
21.0 & 21.0 & -0.101 & 0.201 & 17.69 & 0.42 & 0.00 \\
\hline
\end{tabular}

Figure 8 shows a comparison between the measured and calculated variations of focal length. They are both positive and correspond to an increase of the lens focal length. The closest results to the measurements are the one obtained from the sum of the two calculated defoci. It shows that the refractive index gradient has an important impact on the focal length modification.

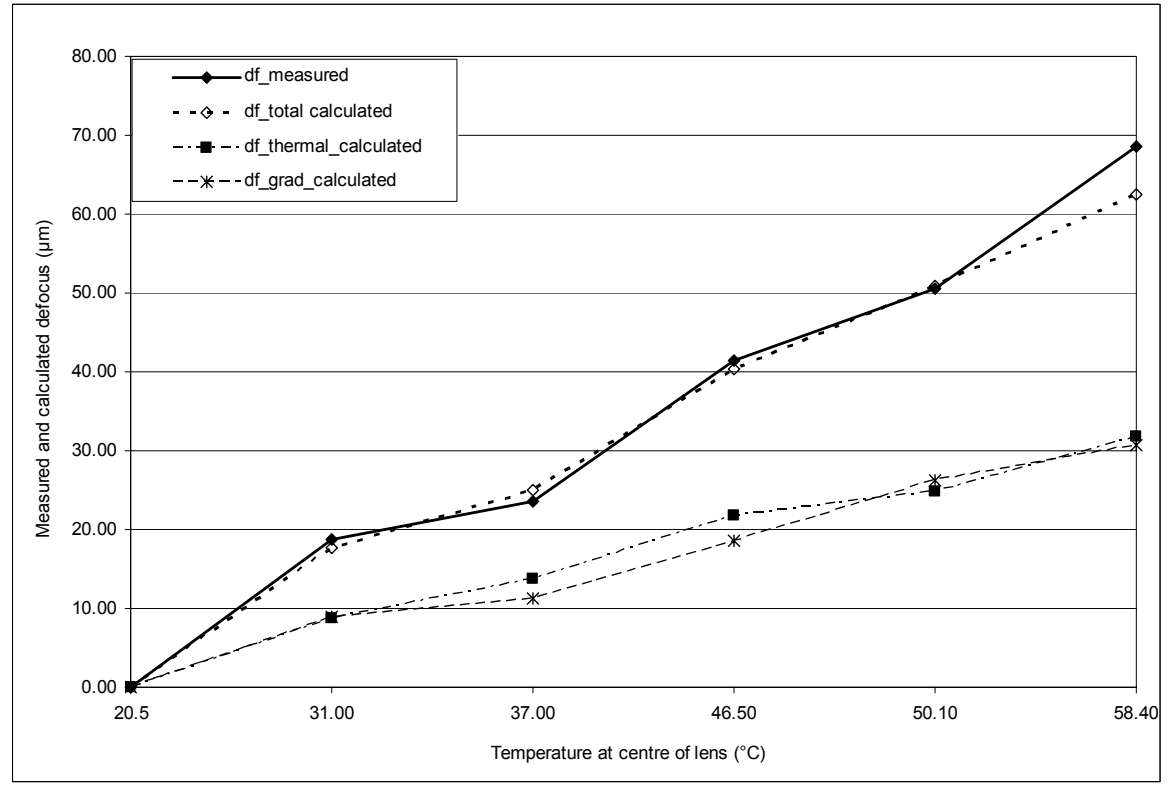

Figure 8. Measured and calculated variations of focal length 


\subsection{YAG bar}

\subsubsection{Interferometric measurements}

The interference that has to be measured here is the one occurring between the reference caliber of the interferometer and the beam that has passed twice inside the YAG bar (by reflection on the plane mirror). A parasite interference with a higher contrast than the one we want to measure appears when the YAG bar is well aligned in the beam. This parasite interference comes from the reflection between the two extreme faces of the bar which are not perfectly parallel. To get rid of this parasite the bar has to be tilted until this interference is no more visible. This small rotation will introduce a small tilt aberration in the results. A picture of the test bench and a view of the interference between the two faces of the bar are shown on Figure 9.
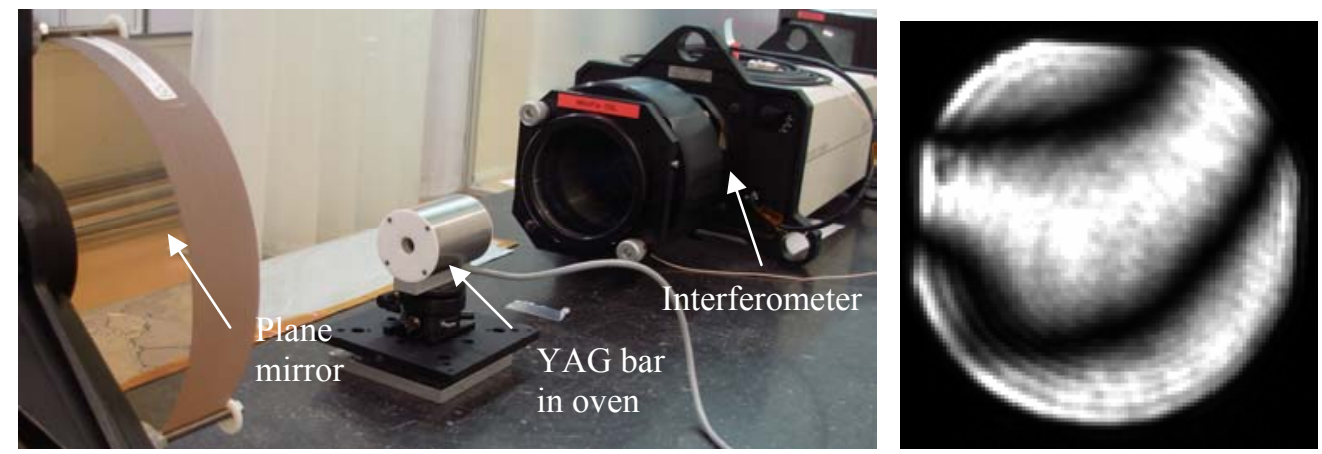

Figure 9. YAG bar interferometric test bench and interference between the 2 extreme faces of the bar

Figure 10 presents three WFE measured respectively at ambient temperature $\left(20^{\circ} \mathrm{C}\right)$ and for an oven temperature of $60^{\circ} \mathrm{C}$ and $100^{\circ} \mathrm{C}$. A stabilization time of half an hour has been considered between the temperature set and the measurement (The results in $\S 4.2 .1$ show that the stabilization is reached after 15 minutes). It can be seen that a focus appears with the temperature (red circular surface in the WFE). The fact that the focus is not centered in the image is probably due to the tilt of the bar. Figure 11 shows the evolution of the RMS WFE and of the focus aberration with the temperature.
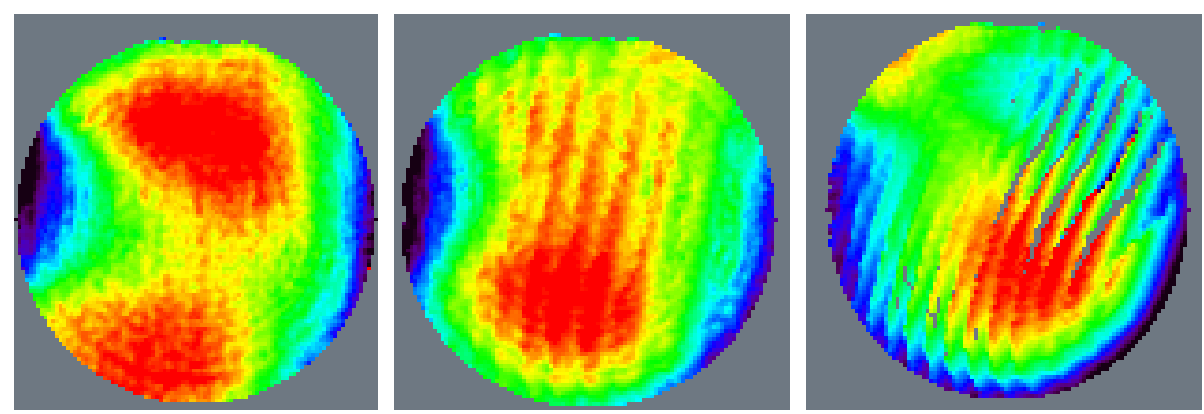

Figure 10. Measured WFE at $\mathrm{T}_{\text {oven }}=20^{\circ} \mathrm{C}, 60^{\circ} \mathrm{C}$ and $100^{\circ} \mathrm{C}$ 


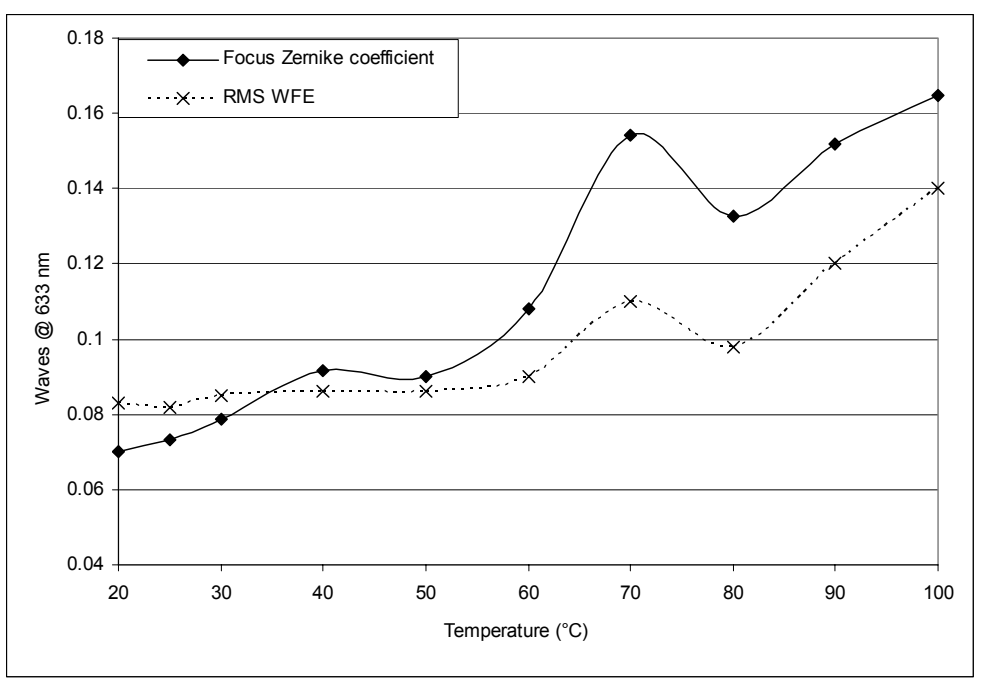

Figure 11. Amplitude of the RMS WFE and focus aberration in function of the temperature

\subsubsection{Polarimetric measurements}

Figure 12 presents the evolution of the phase shift between TE and TM polarizations in function of the applied temperature. It shows that a small birefringence appears with increasing temperature. Figure 13 presents the temporal evolution of the light intensity passing through the YAG bar placed between two crossed polarizers. This experiment is realized during a heating phase between two temperature cases. An increase of intensity measured by the detector means a modification of the polarization state in the YAG bar, due to a thermally induced birefringence in this case.

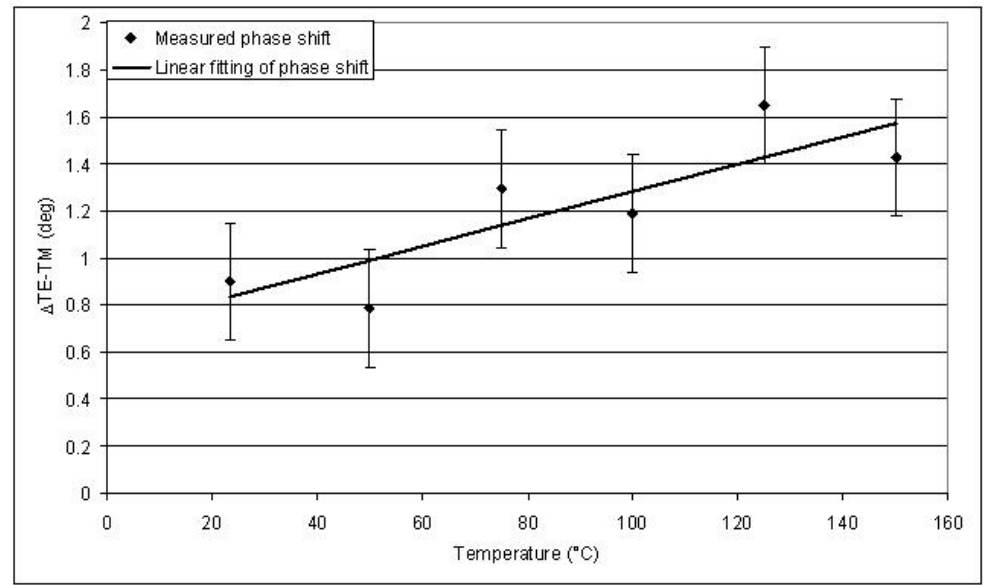

Figure 12: Evolution of the phase-shift between TE and TM polarization in function of YAG-bar temperature.

In Figure 13, two main regions are distinguishable. The first one is associated to the heating phase when the second one is the temperature stabilization. During the heating phase a significant increase of laser light passing through the crossed polarizers and the YAG bar is measured. This means that a polarization rotation or depolarization occurs inside the bar. This effect is certainly due to the birefringence induced inside the bar because of the temperature gradient. After the stabilization phase (around 15 minutes) the birefringence comes back close to its initial value. 


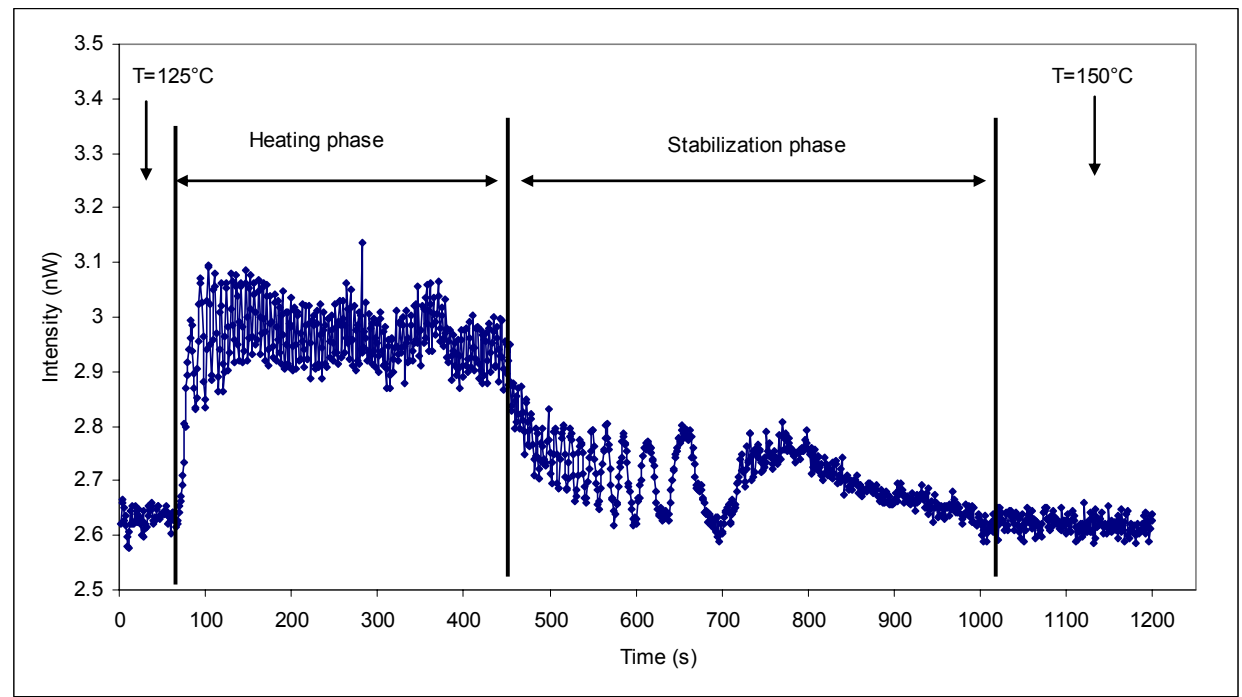

Figure 13: Evolution of the intensity during heating phase of the light transmitted through the YAG and two crossed polarizers.

\section{SIMULATION RESULTS}

\subsection{Spherical lens}

The model of the lens and its mount is shown in Figure 14. The fixation rods, shown in Figure 1, are not modeled since they have a negligible influence on the lens deformation.

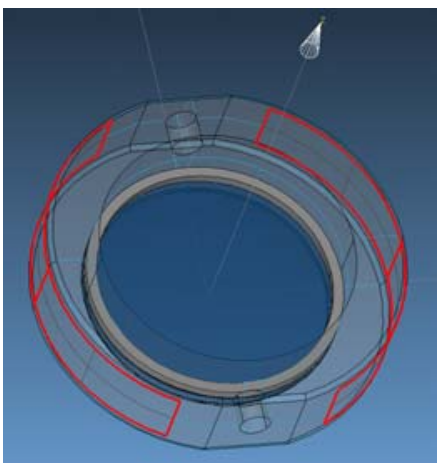

Figure 14. View of the model of the lens and its mount in SAMCEF Field

Appropriate contacts between the lens, the barrel and the maintaining ring are defined. The contacts between the lens and the barrel and between the lens and the ring are limited to wires. Since the heating of the lens may lead to a loss of these contacts (partial or total), two simulations are considered. The first model involves thermo-mechanical wire to surface contacts. It does not permit any slide motion of the lens in its mount. The second model uses thermal wire to surface contacts. It lets the lens free in order to simulate the case of contact loss between the lens and its mount, while insuring the thermal load. One may expect that the right configuration is between these two extreme behaviors.

The measured temperatures are applied on the periphery of the mount in order to simulate the applied heating. Convective fluxes between the lens and ambient air are also taken into account. Both models lead to the same temperature field. The simulated temperatures inside the lens are in good accordance with the experimental values presented in $\S 4.1$, as shown in Figure 15. 


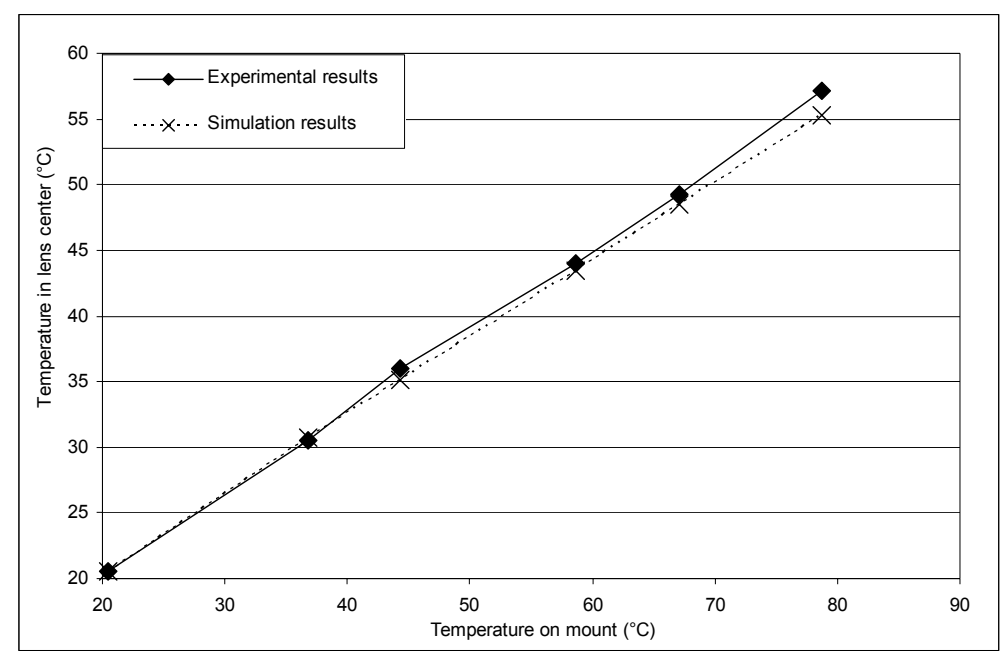

Figure 15. Comparison of simulated and measured temperatures in the center of the lens, versus applied temperature

Even if the temperature gradient is the same for both models, the deformations of the lens and its mount are quite different for the simulation 1 (thermo-mechanical wire to surface contacts) compared with the simulation 2 (thermal wire to surface contacts). In the second model, the lens is free to deform as if no mount was present. The comparison between the two cases is shown in Figure 16.

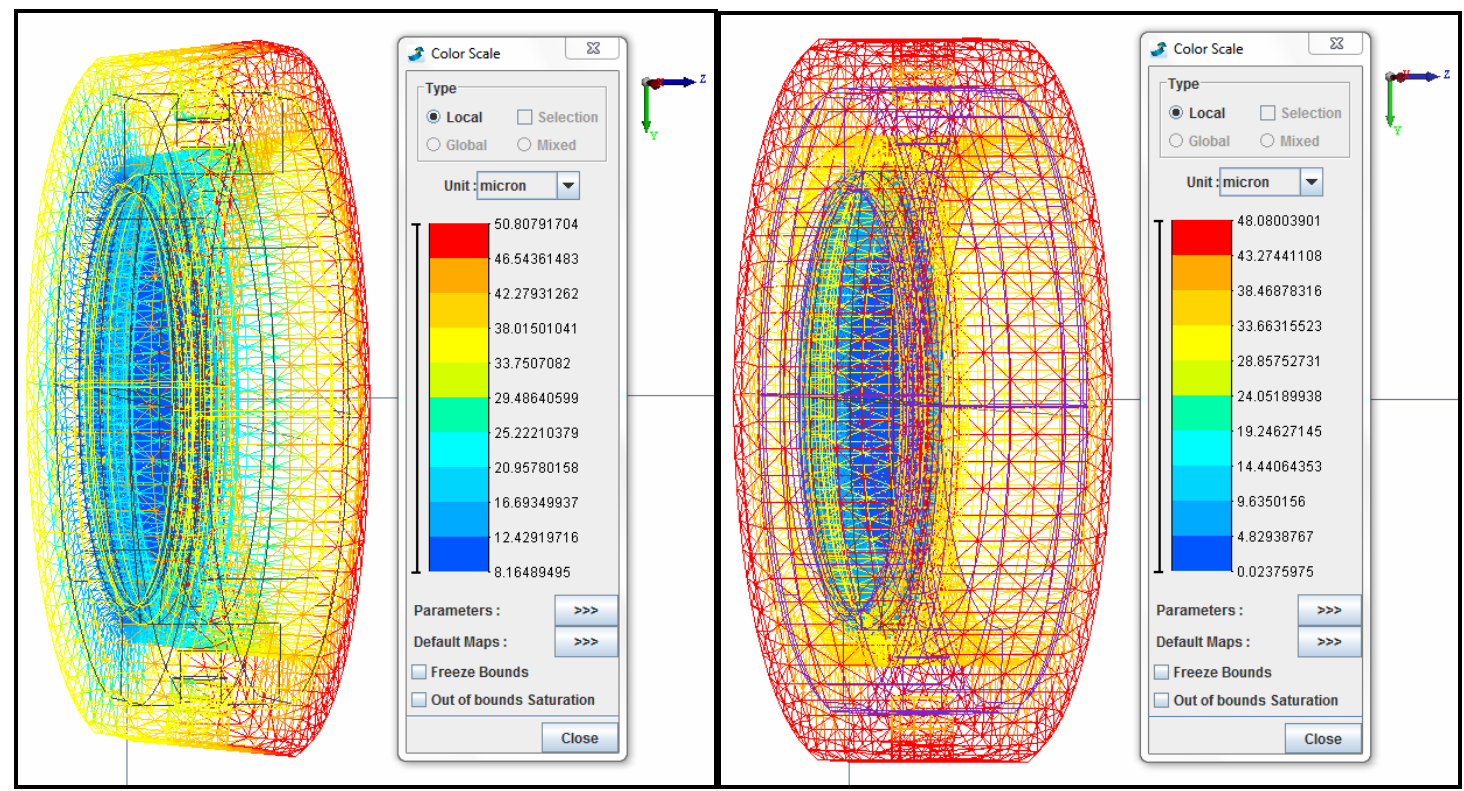

Figure 16. Comparison between the two studied model deformations (simulation 1 on left, simulation 2 on right)

The thermally induced degradation of optical performances is evaluated from the deformation of the two optical surfaces of the lens and the refractive index change. These data are computed by OOFELIE and automatically exported to ZEMAX at the end of the computation. Figure 17 illustrates the simulation results, compared with the experimental values. This comparison is based on the focus aberration, i.e. the $Z_{2,0}$ Zernike standard coefficient, computed by ZEMAX. Again, the results of the two simulations are given. The second model leads to a better accordance with experimental measures. 


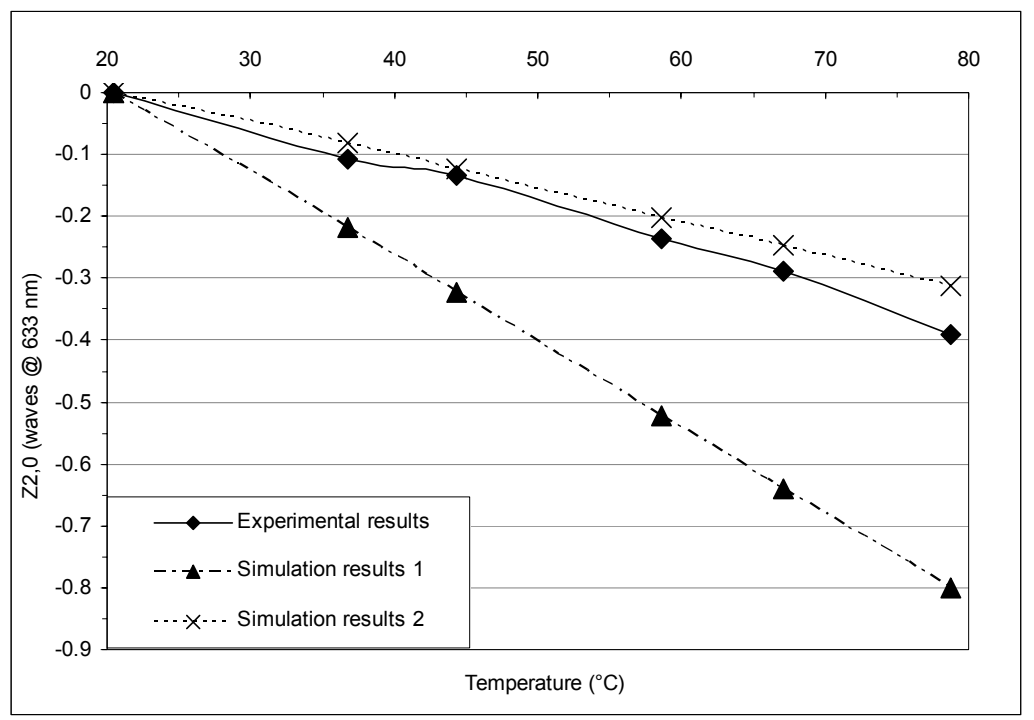

Figure 17. Comparison of simulated and measured focus aberration

The above results are obtained by ray-tracing in the optical software ZEMAX. Both surface deformation and refractive index gradient (GRIN) are exported to ZEMAX. An example of GRIN profile is illustrated in Figure 18, while Figure 19 shows the exported data in ZEMAX. A user defined surface is used for exporting the surface deformation and the GRIN simultaneously. This "OOZerGrin" surface type is provided by Open Engineering. The surface deformation is fitted by Zernike standard polynomials and the GRIN is approximated by a quadratic polynomial interpolation. A dummy surface has to be introduced in the ZEMAX model between the two lens surfaces. It does not give rise to any error in the wavefront aberration coefficients. Nevertheless care has to be taken when surface deformations of higher magnitude than the one observed here are introduced in the optical model.

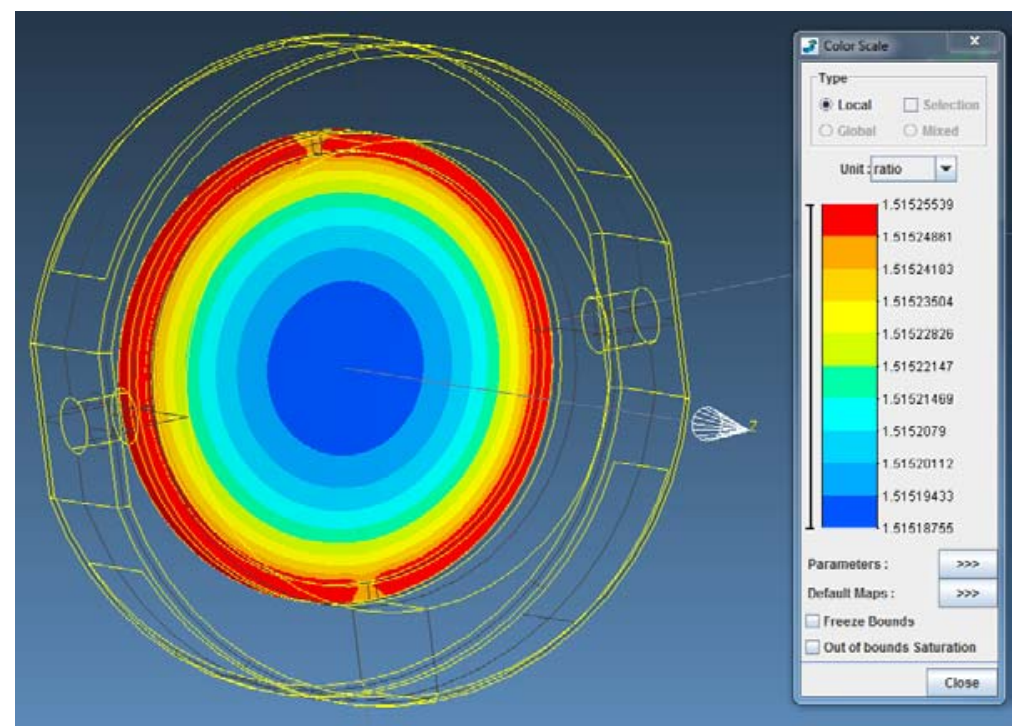

Figure 18. Refractive index gradient in the lens for an applied temperature of $78.7^{\circ} \mathrm{C}$ 


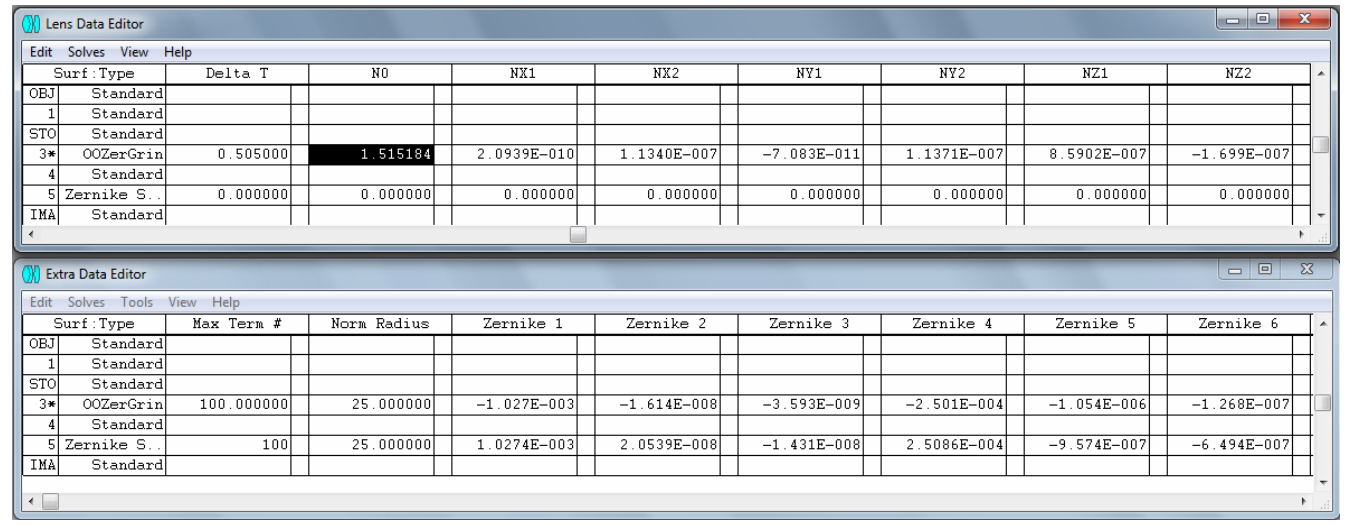

Figure 19. Exportation in ZEMAX of surface deformations and refractive index gradient (applied temperature of $78.7^{\circ} \mathrm{C}$ )

Because of the small surface deformations the impact of the refractive index change is quite important. In the initial optical model defined in ZEMAX, the refractive index was set to 1.51509 (BK7 glass at ambient temperature of $20^{\circ} \mathrm{C}$ and at the operating wavelength of $633 \mathrm{~nm}$ ). The GRIN computation by OOFELIE is realized by taking into account only the $\mathrm{dn} / \mathrm{dT}$ parameter $\left(2.8 \mathrm{e}-6{ }^{\circ} \mathrm{C}^{-1}\right.$ for the $\mathrm{BK} 7$ glass $)$. It could be improved by adding more thermo-optic coefficients.

The presented simulation results are in good agreement with the measured values of the focus aberration. The best accordance is obtained with the model 2, i.e. in the case where thermal but no mechanical contacts between the lens and its mounts are defined. As expected, the experimentally observed defocus versus temperature is between the two simulated tendencies. These results show that the contact is -at least partially- lost. Further improvement could be achieved by the development in OOFELIE of wire to surface mechanical contacts which would authorize some sliding between the wire and the surface while keeping the normal nodal displacements equal. This kind of contact already exists in OOFELIE for surface to surface gluing.

\subsection{YAG bar}

The numerical model of the YAG bar does not take into account the fixation of the bar inside the oven. The oven is not modeled. The experimental applied temperatures are imposed on the faces in contact with the oven. On the two other faces, i.e. the entrance and exit faces of the YAG bar, convective fluxes are defined to simulate heat exchanges with ambient air. The thermal lensing effect is evaluated from the deformation of these two surfaces and from the refractive index change. Figure 20 presents the simulated focus aberrations $\left(\mathrm{Z}_{2,0}\right.$ Zernike standard coefficient) versus applied temperatures, and the experimental data for comparison.

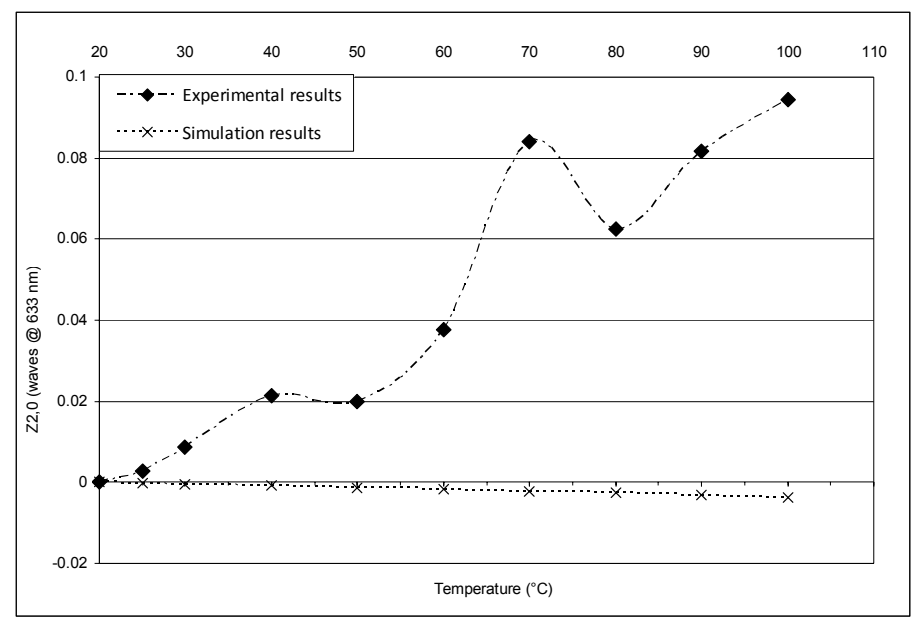

Figure 20. Comparison of simulated and measured focus aberration 
Figure 21 shows the change of refractive index due to heating. The temperature maps (not presented here) are identical since the thermo-optic effect is modeled through a linear approximation by use of the $\mathrm{dn} / \mathrm{dT}$ parameter $\left(7.3 \mathrm{e}-6^{\circ} \mathrm{C}^{-1}\right.$ for $\mathrm{Nd}: \mathrm{YAG}$ ). The initial refractive index is fixed to 1.83 (corresponding to the ambient temperature of $20^{\circ} \mathrm{C}$ and the operating wavelength of $633 \mathrm{~nm}$ ). As shown in Figure 21, the simulated refractive index gradient arising near the two extreme faces of the bar has a very small amplitude, while the global increase of refractive index is more significant. That is why the focus aberration computation does not account for the refractive index gradients at the entrance and exit of the YAG bar. Only global refractive index change is taken into account.
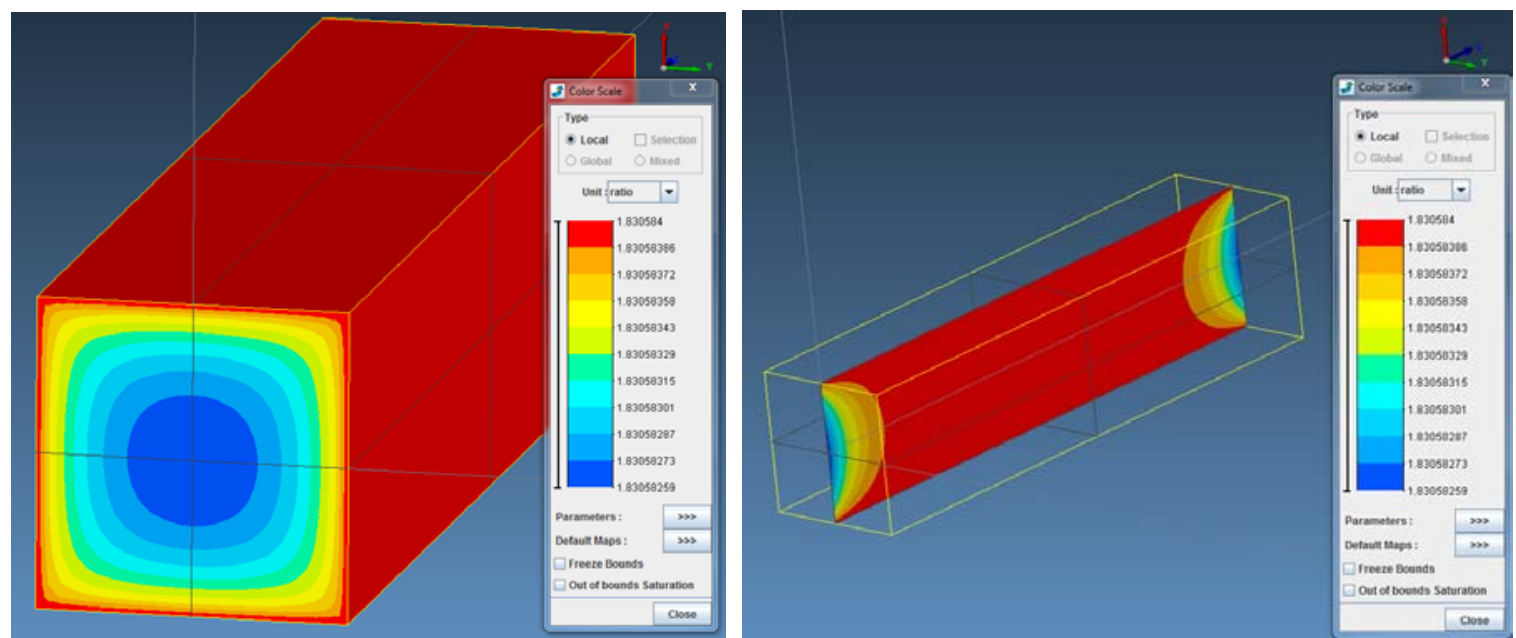

Figure 21. Simulated refractive index change for an applied temperature of $100^{\circ} \mathrm{C}$

These preliminary results show that the simulated thermal lensing is far below the measured one. The focus aberration numerical results are more than one order of magnitude below the measured values. All simulation results given here correspond to static equilibrium solutions. We can expect that experimental temperature gradients were stronger than the simulated ones, leading to stronger surface deformations and stronger refractive index gradients. Both numerical simulation and experimental characterization have thus to be upgraded. Ideally, the thermal strain-induced birefringence should be taken into account for simulating the lensing effect in a laser bar. Still under development, the optical anisotropy computed by OOFELIE is not linked to ZEMAX. Furthermore, the definition of the thermal loads in the numerical model could be improved. This will lead to other simulated temperature distributions in the YAG bar which could be compared with experimental data. The simulation of the oven and the consideration of radiative exchanges between the oven and the bar are also planned.

The studied Nd:YAG bar has its optical axis in the crystallographic [111] direction (growth axis). With the cubic symmetry of the crystal in this configuration, the strain-induced birefringence is characterized by three parameters which composed the strain-optic matrix $\left(\mathrm{p}_{11}=-0.0290, \mathrm{p}_{12}=0.0091, \mathrm{p}_{44}=-0.0615\right)$. Since experimental measurements and numerical solutions have to be completed, the birefringence simulation results presented in this paper are compared with theoretical values. This is given in Figure 22 in the case of a cylinder Nd:YAG laser rod operating at the wavelength of $1.06 \mu \mathrm{m}$. The pumping power is $4 \mathrm{~kW}$ with a thermal absorption rate of $5 \%$. In accordance with the theoretical modeling ${ }^{8}$, the optical path difference between two crossed linearly polarized plane waves is proportional to the square of the radial coordinate of the traveling rays and is independent of the angular coordinate. The impact of thermo-optic effect in the birefringence computation is negligible in that case, as shown in Figure 22. Indeed, the first simulation (Simulation 1) is obtained by considering a null value of $\mathrm{dn} / \mathrm{dT}$, while the second one (Simulation 2) accounts for thermo-optic effect. The result of Figure 22 shows a very good accordance between theoretical and simulated thermally induced birefringence, which is computed from the thermo-mechanical strain distribution in the YAG bar and the knowledge of the strain-optic coefficients. 


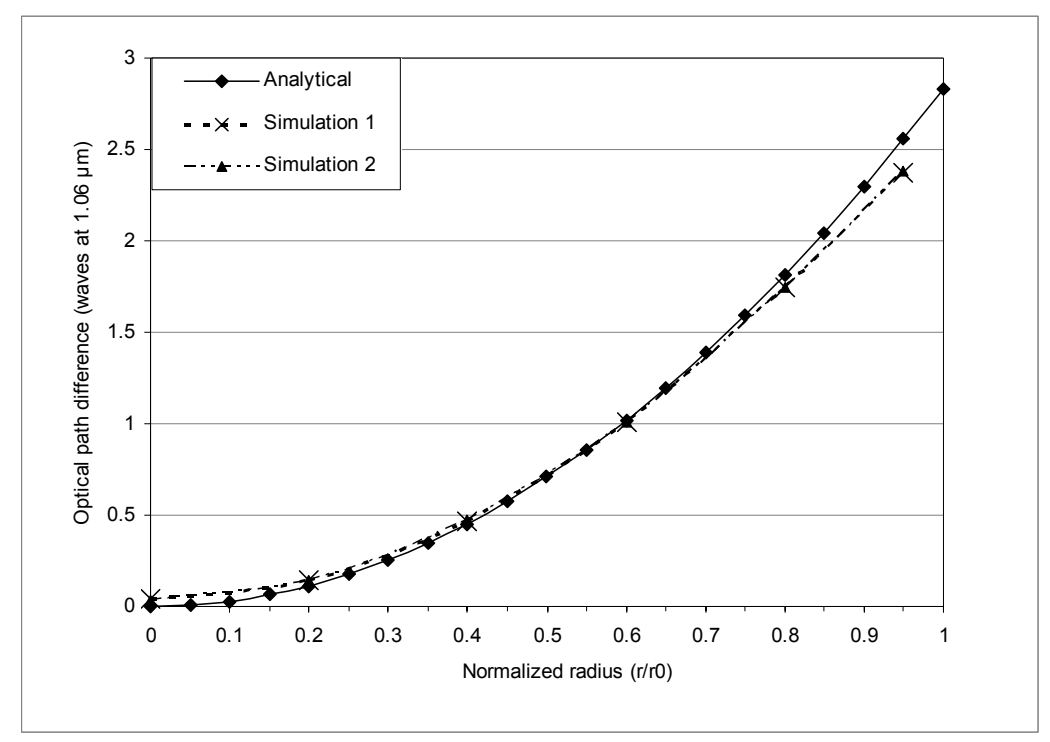

Figure 22. Comparison of simulated and theoretical optical path differences

\section{CONCLUSIONS}

In order to confront the simulation results obtained by OOFELIE Multiphysics with experimentations two test benches have been implemented. The first one consists in interferometric measurements of a spherical lens in a barrel at different temperatures between $20^{\circ} \mathrm{C}$ and $80^{\circ} \mathrm{C}$. The second test bench includes interferometric and polarimetric measurements of a YAG bar heated in a dedicated oven (from $20^{\circ} \mathrm{C}$ to a maximum of $200^{\circ} \mathrm{C}$ ).

The interferometric measurements of the lens showed good agreement between the experimental and simulated WFE degradation. The comparison has been performed through the Zernike coefficient of the focus aberration which is the dominant effect of the heating. The impact on the lens optical performances is a modification of its focal length. Two simulation models have been considered. The first one involves thermo-mechanical wire to surface contacts. It does not permit any slide motion of the lens in its mount. The second model used thermal wire to surface contacts. It lets the lens free in order to simulate the case of contact loss between the lens and its mount, while insuring the thermal load. The model which gave the best match with the experiment is the second one.

For the YAG bar the simulations of thermal lensing were not compliant with the measurements. Effects such as the thermal strain-induced birefringence, a better definition of the thermal loads and the consideration of radiative exchanges between the oven and the bar should be part of some improvements to the model.

Concerning the polarimetric measurements of the YAG bar only preliminary experiments have been carried out to highlight the presence of some birefringence inside the bar due to a change of temperature. It was shown that the birefringence appears during the heating phase of the oven then decreases with some damping during the stabilization phase to finally come back to its initial value (or very close to it) when the equilibrium is reached. These preliminary experimental results could not be used for comparison with the simulation results obtained with OOFELIE Multiphysics. That's why analytical results have been considered and a good agreement between the theoretical and simulated thermally induced birefringence has been observed.

In the context of the Multi- $\Phi$ project, several recent advances in simulation techniques are integrated in OOFELIE Multiphysics. Thanks to these software improvements, concerning opto-thermo-mechanical design, fluid-structure interaction and other multiphysics applications such as aero-vibro-acoustics or piezoelectric actuators, efficient simulations of complex industrial applications are expected. 


\section{ACKNOWLEDGMENT}

The present work has been conducted in the context of the Multi- $\Phi$ project, funded by the Marshall Plan of the Walloon Region (Belgium), which is acknowledged by the authors.

\section{REFERENCES}

[1] Klapka I. and Cardona A., "An object Oriented implementation of the finite element method for coupled problem », Revue Européenne des Eléments finis, vol. 7, n 5, 469-504 (1998).

[2] Partenov V., Shashkin V. and Stepanov E., « Numerical Investigation of Thermally Induced Birefringence in Optical Elements of Solid-State Lasers », Appl. Opt., vol. 32, n 27, 5243-5255 (1993).

[3] Nye, J.F., [Physical Properties of Crystals - Their Representation by Tensors and Matrices], Clarendon Press, Oxford (2009).

[4] Jamieson, T.H., « Athermalization of optical instruments from the optomechanical viewpoint », SPIE Proc., CR43 (1992).

[5] Yoder,P.R., Jr., [Mounting Optics in Optical Instruments], SPIE Press (2002).

[6] Jamieson, T.H., « Thermal effects in optical systems », Opt. Eng. 20 (April 1981).

[7] Foster J.D. and Osterink L.M., "Thermal Effects in a Nd :YAG Laser », J. Appl. Phys. 41(9), 3656-3663 (1970).

[8] Koechner W. and Rice D.K., «Effect of Birefringence on the Performance of Linearly Polarized YAG:Nd Lasers », IEEE J. Quantum Electron. QE-6(9), 557-566 (1970).

[9] Koechner W., « Thermal Lensing in a Nd:YAG Laser Rod », Appl. Opt. 9(11), 2548-2553 (1970). 